\title{
Treatment of fusiform ascending aortic aneurysms: A comparative study with 2 options
}

\author{
Hao Zhang, MD, Fanglin Lu, MD, Dan Qu, MD, Lin Han, MD, Jibin Xu, MD, Guangyu Ji, MD, and \\ Zhiyun Xu, MD
}

\begin{abstract}
Objective: Ascending aortic replacement and reinforced reduction aortoplasty are 2 optional procedures for the treatment of fusiform ascending aneurysms. This study was designed to compare the early and late results of these 2 options.
\end{abstract}

\begin{abstract}
Methods: Between January 2000 and January 2008, 71 patients with fusiform ascending aortic aneurysms and aortic valve disease underwent reinforced reduction aortoplasty associated with aortic valve replacement (RRA group, $\mathrm{n}=32$ ) or ascending aortic replacement combined with aortic valve replacement (AAR group, $\mathrm{n}=39$ ). Patients requiring other concomitant cardiac procedures were excluded. Perioperative events and late results were compared.
\end{abstract}

Results: The variables of the 2 groups were similar, except age and preoperative diameter of the ascending aorta. Despite the nearly identical perioperative morbidity in the 2 groups, mean cardiopulmonary bypass time and aortic crossclamping time were shorter in the RRA group. The follow-up period was between 1 and 8 years (mean, 3 years and 4 months). The 5-year survival rate was $90.7 \% \pm 6.4 \%$ versus $87.0 \% \pm 6.3 \%$, respectively. Although there was a significant increase in aortic sinus diameters in the AAR group, all aortic sinus diameters were within the acceptable range. There was no increase in proximal aortic arch diameters in the 2 groups.

Conclusions: For the treatment of fusiform ascending aortic aneurysms, both procedures can result in favorable and comparable late results in appropriate patients. Furthermore, reinforced reduction aortoplasty should be encouraged more because of its significant operative simplicity and safety if only the quality of the aortic wall is acceptable. (J Thorac Cardiovasc Surg 2011;141:738-43)

In modern cardiovascular surgery it remains controversial whether reinforced reduction aortoplasty (RRA) or ascending aortic replacement (AAR) should be applied to patients with fusiform ascending aortic aneurysms. 1,2

Reduction aortoplasty is generally considered a conservative method $^{2}$ because the native endothelium-lined aorta is preserved and surgical invasion is relatively reduced. ${ }^{3-5}$ However, risk of redilatation, dissection, or rupture of the ascending aorta has been reported in long-term follow-up., In consideration of this, it has been strongly recommended that external reinforcement with a Dacron vascular prosthesis should be applied for all reduction aortoplasty procedures. ${ }^{1}$ In contrast, AAR has been considered a radical procedure that demonstrates satisfactory long-term results, ${ }^{7,8}$ although resulting in more surgical risks in the early postoperative period, ${ }^{7,9}$ and might play a role as a risk factor for the development of aortic root dilatation ${ }^{10}$ by increasing wall

From the Division of Cardiothoracic Surgery, Changhai Hospital, Second Military Medical University, Shanghai, China.

Disclosures: Authors have nothing to disclose with regard to commercial support.

Received for publication Dec 31, 2009; revisions received March 21, 2010; accepted for publication May 17, 2010; available ahead of print June 28, 2010.

Address for reprints: Zhiyun Xu, MD, Division of Cardiothoracic surgery of Changhai

Hospital, Second Military Medical University, 168 Changhai Rd, Shanghai, China

(E-mail: zhiyunx@hotmail.com).

$0022-5223 / \$ 36.00$

Copyright (c) 2011 by The American Association for Thoracic Surgery doi:10.1016/j.jtcvs.2010.05.031 tension in the residual aorta. ${ }^{11}$ Based on conflicting standpoints in the literature, the controversy of repair or replacement persists to date. To address this confusing clinical issue, we designed this study to compare the perioperative events and late results of RRA and AAR.

\section{MATERIALS AND METHODS}

We retrospectively reviewed the database for the Division of Cardiothoracic Surgery at the Second Military Medical University and identified all patients undergoing either aortic valve replacement (AVR) with RRA or AVR with AAR between January 2000 and January 2008. The patients who underwent other concomitant cardiac procedures were therefore excluded from this study. None of the patients had Marfan syndrome or aortic dissection. The aortic aneurysm was fusiform in all patients without aortic root involvement.

A total of 71 patients were available for the final analysis. Preoperative demographic characteristics, operative variables, and postoperative and follow-up results were retrieved from the database. In 32 patients (the RRA group) AVR was performed with RRA. In the remaining 39 patients (the AAR group), AVR was performed with concomitant AAR.

\section{Surgical Technique}

Surgical procedures were performed by the members of the Division of Cardiothoracic Surgery at the Second Military Medical University. The choice between 2 procedures was made preoperatively according to integrated evaluation of the following factors: quality of the ascending aortic wall, size of the aneurysm, and operative risk, as well as age.

All procedures were carried out under median sternotomy, cardiopulmonary bypass, and moderate hypothermia, with cardioplegic arrest for myocardial protection. The aortic cannula was placed in the proximal aortic arch or 


\section{Abbreviations and Acronyms}

$\mathrm{AAR}=$ ascending aortic replacement

$\mathrm{AVR}=$ aortic valve replacement

$\mathrm{BAV}=$ bicuspid aortic valve

$\mathrm{RRA}=$ reinforced reduction aortoplasty

femoral artery. The aortic valve was replaced first in all cases. Briefly, an incision was made in the ascending aorta after crossclamping through a longitudinal aortotomy beginning below the aortic clamp and extending into the noncoronary sinus in the patients undergoing RRA or through a transverse incision above the sinuses in the patients undergoing AAR. After cardioplegic arrest was achieved, the aortic valve was excised and replaced with a mechanical prosthesis or bioprosthesis. The aortoplasty was performed by means of removal of an elliptical portion of the dilated aortic wall along the aortotomy incision, as described by Robicsek and Thubrikar. ${ }^{3}$ The patient was weaned from cardiopulmonary bypass after the aortotomy was closed with 4-0 Prolene double-running sutures. A Dacron tube graft with a diameter of approximately 28 to $30 \mathrm{~mm}$ for wrapping of the ascending aorta was cut longitudinally, and 2 pieces of the prosthesis were excised from the ends of the graft. The reduced aorta was wrapped with the formed prosthesis thereafter. The wrap was stabilized by both distal anchoring to the wall of the aortic arch and proximal anchoring to the suture ring of the valve prosthesis and the aortic wall before closure with a running nonabsorbable suture.

AAR was performed with an approximately 28 - to $30-\mathrm{mm}$ Dacron tube graft replacing the dilated ascending aorta above the level of the sinuses.

\section{Follow-up}

Follow-up information was obtained by means of retrospective review of medical records or by means of postal questionnaire and telephone interviews. The diameters of the aorta were all retrieved from computed tomographic scans preoperatively, postoperatively, and during the follow-up period. The measurement was made at the level of bifurcation of the pulmonary artery, aortic sinuses, and proximal aortic arch, respectively.

\section{Statistical Methods}

Categorical variables are expressed as percentages, and continuous variables are expressed as means \pm standard deviations throughout the article. Comparison of categorical variables was performed with the $\chi^{2}$ test, and continuous variables were analyzed with the Student's $t$ test. Comparisons of aortic diameters were analyzed by means of analysis of variance for repeated measures, and Bonferroni's method was used for multiple comparisons. Long-term survival was analyzed with the methods of Kaplan-Meier. Comparisons with groups were made by using the log-rank test. Cox regression was used to identify factors associated with late death.

\section{RESULTS}

\section{Preoperative Demographics}

The preoperative demographics of the study groups are shown in Table 1. Patients undergoing RRA were significantly older than those undergoing AAR, who were more likely to have larger ascending aortic diameters. There were no significant differences in the other variables between the 2 groups.

\section{Operative and Postoperative Details}

The operative and postoperative details for the 2 groups are listed in Table 2. There were no operative deaths in either group. The mean cardiopulmonary bypass and aortic
TABLE 1. Preoperative and intraoperative demographics

\begin{tabular}{lccc}
\hline \multicolumn{1}{c}{ Variable } & $\begin{array}{c}\text { RRA group } \\
(\mathbf{n = 3 2 )}\end{array}$ & $\begin{array}{c}\text { AAR group } \\
(\mathbf{n = 3 9 )}\end{array}$ & $\begin{array}{c}\boldsymbol{P} \\
\text { value }\end{array}$ \\
\hline Age (y) & $56.7 \pm 9.2$ & $51.3 \pm 11.6$ & .04 \\
Male sex & $17(53 \%)$ & $29(74 \%)$ & .06 \\
LV ejection fraction (\%) & $59.9 \pm 6.1$ & $61.2 \pm 6.5$ & .37 \\
NYHA class III or IV & $6(16 \%)$ & $7(10 \%)$ & .72 \\
Diabetes mellitus & $2(6 \%)$ & $2(5 \%)$ & .84 \\
Hypertension & $6(19 \%)$ & $5(13 \%)$ & .49 \\
Bicuspid aortic valve & $13(41 \%)$ & $21(54 \%)$ & .27 \\
Diameter of ascending aorta $(\mathrm{mm})$ & $47.9 \pm 4.0$ & $54.3 \pm 5.3$ & $<.001$ \\
Diameter of aortic sinuses (mm) & $30.4 \pm 0.9$ & $30.6 \pm 1.0$ & .53 \\
Diameter of proximal & $28.9 \pm 1.0$ & $29.3 \pm 1.1$ & .20
\end{tabular}

aortic $\operatorname{arch}(\mathrm{mm})$

$R R A$, Reinforced reduction aortoplasty; $A A R$, ascending aortic replacement; $L V$, left ventricular; NYHA, New York Heart Association.

crossclamping times in the RRA group were shorter than those in the AAR group $(82 \pm 14$ vs $113 \pm 19$ minutes $[P<.001]$ and $42 \pm 10$ vs $71 \pm 20$ minutes $[P<.001]$, respectively). Less red blood cells were used for patients in the RRA group than in the AAR group $(3.5 \pm 1.7$ vs $4.8 \pm 2.1$ units, $P=.01)$. The total in-hospital stay of the RRA group was significantly shorter than that of the AAR group (14.4 \pm 3.8 vs $17.2 \pm 4.4$ days, $P=.01$ ). Intra-aortic balloon support was not used in all patients. There were no statistically significant differences in rates of perioperative re-exploration for bleeding, respiratory insufficiency, stroke, or renal dysfunction between the groups $(P>.05)$.

\section{Late Results}

The follow-up period was between 1 and 8 years (mean, 3 years and 4 months). The follow-up was complete in all patients. Three patients in the RRA group and 5 patients in the AAR group died during late follow-up. A total of 63 patients were alive at the time of the last follow-up examination.

TABLE 2. Operative and postoperative details

\begin{tabular}{lccc}
\hline \multicolumn{1}{c}{ Variable } & $\begin{array}{c}\text { RRA group } \\
(\mathbf{n}=\mathbf{3 2})\end{array}$ & $\begin{array}{c}\text { AAR group } \\
(\mathbf{n = 3 9 )}\end{array}$ & $\begin{array}{c}\boldsymbol{P} \\
\text { value }\end{array}$ \\
\hline CBP time (min) & $82 \pm 13$ & $104 \pm 16$ & $<.001$ \\
Aortic crossclamp time (min) & $43 \pm 9$ & $67 \pm 15$ & .001 \\
Total pRBCs (unit) & $3.5 \pm 1.7$ & $4.8 \pm 2.1$ & .01 \\
IABP support & 0 & 0 & - \\
Mortality & 0 & 0 & - \\
Re-exploration for bleeding & $2(6.2 \%)$ & $3(7.7 \%)$ & .81 \\
Prolonged ventilation & $1(3.1 \%)$ & 0 & .26 \\
Stroke & $1(3.1 \%)$ & $1(2.6 \%)$ & .89 \\
Renal dysfunction & $2(6.2 \%)$ & $2(5.1 \%)$ & .84 \\
ICU stay (d) & $2.2 \pm 1.5$ & $3.0 \pm 1.9$ & .05 \\
Total in-hospital stay (d) & $14.4 \pm 3.8$ & $17.2 \pm 4.4$ & .01 \\
\hline
\end{tabular}

$R R A$, Reinforced reduction aortoplasty; $A A R$, ascending aortic replacement; $C P B$, cardiopulmonary bypass; $p R B C s$, packed red blood cells; $I A B P$, intra-aortic balloon pump. 


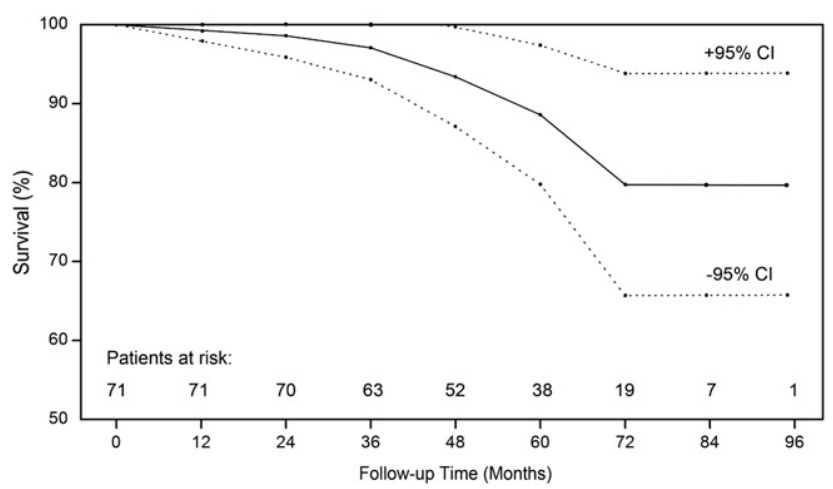

FIGURE 1. Overall survival of patients in the whole series (Kaplan-Meier curve $\pm 95 \%$ confidence interval). Numbers of patients at risk at each time point are noted. RRA, Reinforced reduction aortoplasty; $A A R$, ascending aortic replacement.

The overall survival rate in all patients was $88.6 \% \pm$ $4.5 \%$ at 5 years (Figure 1). The 5-year survival rate was $90.7 \% \pm 6.4 \%$ in the RRA group and $87.0 \% \pm 6.3 \%$ in the AAR group (Figure 2). There was no difference in the survival of the patients in the 2 groups $(P=.59)$.

There were 2 cardiac-related deaths during follow-up, with myocardial infarction in 1 patient of the RRA group and sudden death in 1 patient of the AAR group. Six noncardiac deaths occurred during follow-up. Two patients died of cancer and traffic accidents in the RRA group. Four deaths in the AAR group were attributed to cancer, renal failure, cerebral bleeding, and pneumonia, respectively.

There were no reoperations on the aorta or aortic valve in all patients during follow-up.

Only age was found to be a significant predictor in determination of late death in each group. The type of aortic procedure was not a significant predictor of late death in the whole cohort $(P=.593)$. Other factors, such as sex, left ventricular ejection fraction, bicuspid aortic valve (BAV), and preoperative diameters of the ascending aorta were not significant predictors in either group. Table 3 outlines variables that were evaluated for their effect on late death by using Cox regression.

\section{Variation of Aortic Diameters}

Both procedures resulted in a significant reduction in ascending aortic diameter (RRA group: $47.9 \pm 4.0$ vs $27.3 \pm$ $0.7 \mathrm{~mm}, P=.000$; AAR group: $54.3 \pm 5.3$ vs $29.5 \pm 0.4$ $\mathrm{mm}, P=.000)$.

The mean diameters of the aortic sinuses and proximal aortic arch in all patients are shown in Figure 3 for each group as a function of time. Thirty-nine patients (18 in the RRA group and 21 in the AAR group) who accumulated at least 60 months of follow-up were further analyzed for assessing late redilatation in the aorta. In these patients there was no difference in the diameters of the aortic sinuses between the postoperative and late follow-up periods in the RRA group ( $30.4 \pm 0.7$ vs $30.8 \pm 1.1 \mathrm{~mm}, P=.097)$. The diameters of the sinuses increased significantly in the AAR group during follow-up (postoperatively: $30.6 \pm 0.8 \mathrm{~mm}$ vs late

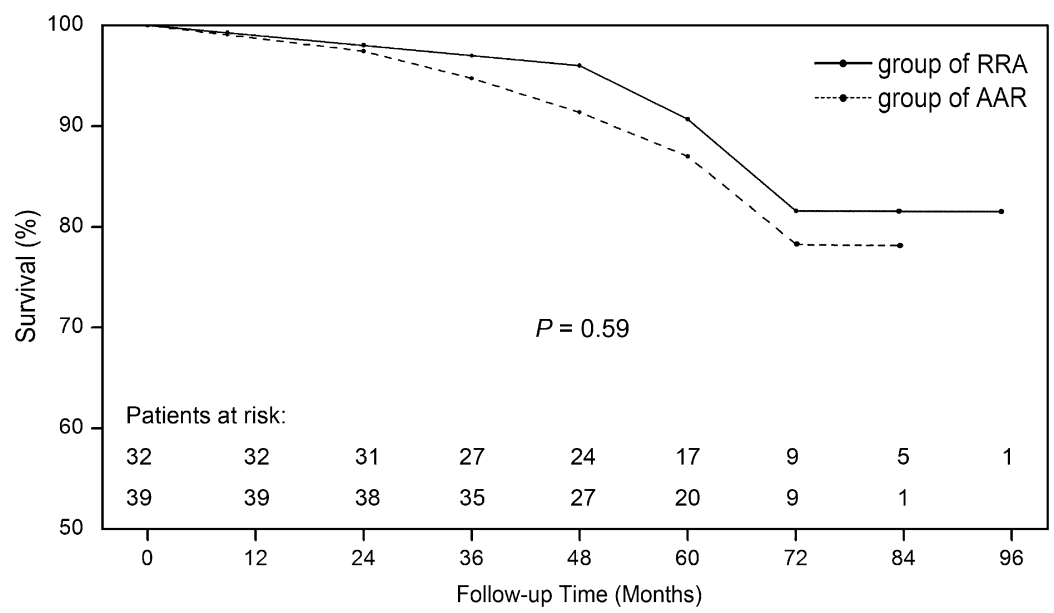

FIGURE 2. Survival of patients in the reinforced reduction aortoplasty $(R R A)$ and ascending aortic replacement $(A A R)$ groups during follow-up. There was no difference in survival between groups. Numbers of patients at risk at each time point are given. 
TABLE 3. Cox regression of variables related to late death

\begin{tabular}{lcc}
\hline & \multicolumn{2}{c}{$\boldsymbol{P}$ value } \\
\cline { 2 - 3 } \multicolumn{1}{c}{ Variable } & RRA group & AAR group \\
\hline Age & .038 & .006 \\
Sex & .937 & .896 \\
EF & .393 & .112 \\
Bicuspid valve & .684 & .506 \\
Preoperative ascending & .149 & .970 \\
$\quad$ aorta diameter & & \\
$R R A$, Reinforced reduction aortoplasty; $A A R$, ascending aortic replacement; $E F$, ejec- \\
tion fraction.
\end{tabular}

follow-up: $31.0 \pm 1.0 \mathrm{~mm}, P=.014)$. However, all diameters of sinuses were within the acceptable range, and no reoperation was required for all patients during the follow-up period. In each group there was no difference in the diameters of the proximal aortic arch between the postoperative period and late follow-up (RRA group: $28.7 \pm 0.8$ vs $29.1 \pm 0.9$ $\mathrm{mm}, P=.06$; AAR group: $29.1 \pm 1.3$ vs $29.3 \pm 1.4 \mathrm{~mm}$, $P=.69)$. No late complications related to recurrent aortic dilatation were identified during follow-up.

\section{DISCUSSION}

Open surgical intervention is still the predominant therapeutic strategy for the treatment of ascending aortic aneurysms in the era of endovascular intervention. ${ }^{12}$ According to the various locations of the aorta impaired by pathological changes, different surgical procedures are indicated. For aneurysms with aortic root involvement, root replacement or reconstruction is indicated. In terms of ascending aortic aneurysms without root involvement (ie, fusiform ascending aortic aneurysm), AAR and RAA are frequently performed procedures. ${ }^{1}$

RAA is generally considered conservative for the preservation of the native aortic wall, whereas AAR is radical because of its surgical invasiveness. ${ }^{3,7}$ Along with progress and refinement in surgical technique, the early surgical results with both options have been significantly improved. ${ }^{2}$

However, RAA without external support was largely abandoned because of its demonstrated high recurrence rate of dilatation. ${ }^{6}$ In recent years, a series of clinical studies have been carried out focusing on the evaluation of RRA. ${ }^{13-16}$ Nevertheless, most of these results of RRA were reported separately without comparison with the AAR procedure. Therefore it would be less valuable to compare the early and late outcomes with existing AAR data.

Another issue in previous studies of RRA is that the observation of postoperative redilatation was mostly focused on the examination of the diameter of the ascending aorta, ${ }^{2,13,16,17}$ whereas the fate of the aortic diameters at the sinuses and distal aorta after RRA is left unknown.

In the latest decade, Egloff and associates ${ }^{4}$ reported that RRA carried a lower complication rate than the other 3 procedures, including unsupported reduction aortoplasty, supracoronary graft replacement, and composite graft replacement. Carrel and coworkers ${ }^{18}$ compared 3 different surgical options for treatment of ascending aortic aneurysms in a heterogeneous group of patients and reported a lowest

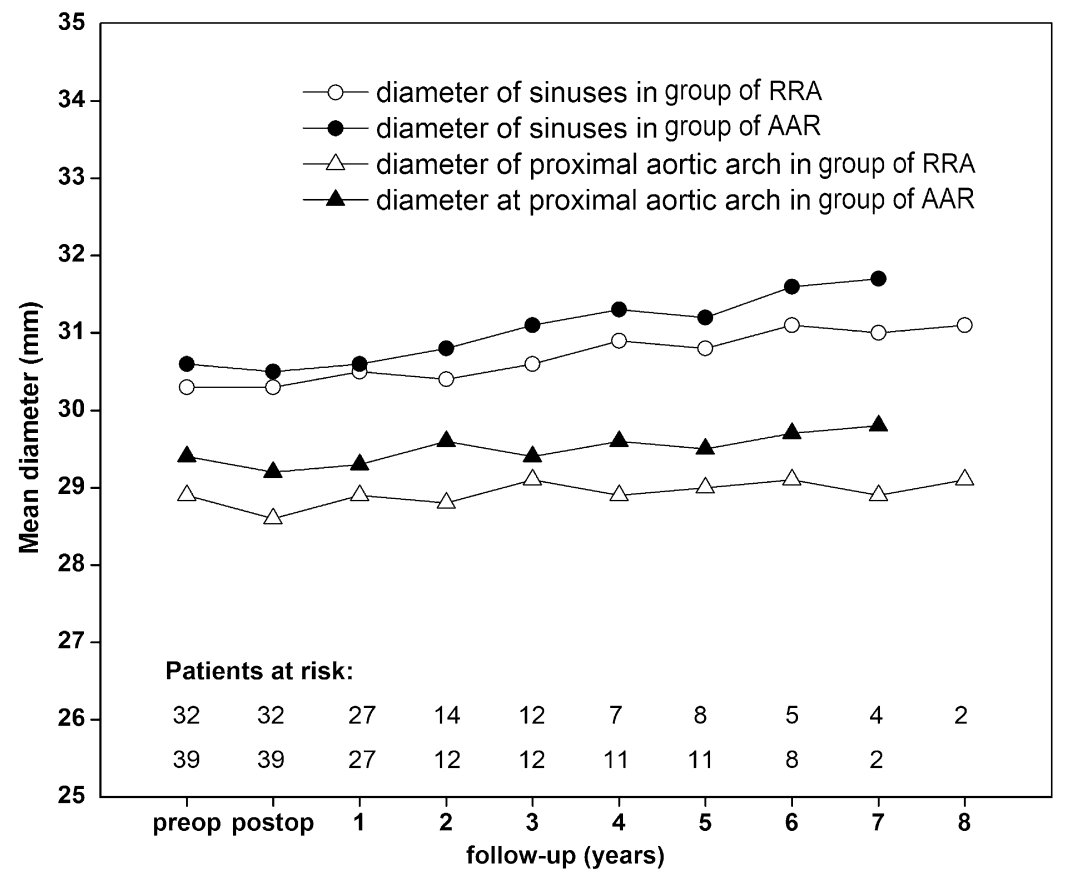

FIGURE 3. Mean diameter of aortic sinuses and proximal aortic arch of each group during follow-up. Numbers of patients at risk at each time point are given. $R R A$, Reinforced reduction aortoplasty; $A A R$, ascending aortic replacement; preop, preoperatively; postop, postoperatively. 
early mortality $(1.8 \%)$ and the best 8 -year survival $(89.6 \%)$ in the reinforced RRA group over supracoronary graft replacement and composite graft.

However, both studies were carried out in heterogeneous cohorts because there were not only patients with ascending aortic aneurysm with aortic root involved but also patients without aortic roots involved. It is well known that ascending aortic aneurysms with or without roots involved indicate different procedures and that the difference in the diseases per se might lead to different outcomes. Therefore the groups of patients in the 2 studies were not truly comparable, which limited the reliability of the results and conclusion.

In addition, different cardiac procedures were often performed in these studies, which might be an interference factor that affects the results significantly. ${ }^{13-16}$

Patients with ascending aortic aneurysms are most commonly combined with aortic valve disease. ${ }^{1,19}$ Polvani and associates ${ }^{15}$ reported a high rate of $88.2 \%$ in their group. We included all patients with fusiform ascending aorta and AVR as the only concomitant procedure to maximize the available cases and minimize the interference to the comparison. For the evaluation of proximal and distal ascending aortic diameters, we focused continuously on the diameters of the aortic sinuses and proximal aortic arch, instead of the ascending aorta, which was already demonstrated not likely to be redilatated with 2 options. ${ }^{8,16}$

Like any decision making in modern cardiovascular surgery, the value of every risk or benefit of the treatment should be considered synthetically for patients with an ascending aortic aneurysm. The factors that were well summarized by Sievers ${ }^{20}$ include size, wall thickness, and pathology of the aorta; hemodynamic load caused by aortic valve pathology; concomitant cardiac surgery; and progression of dilatation. Moreover, life expectancy and individual experience of the surgeon also contribute to decision making.

Of these, the size of the ascending aorta and the morphology of the aortic wall are usually considered the most important determinants for the surgical intervention. ${ }^{16}$ Elefteriades ${ }^{21}$ suggested that intervention for the ascending aortic aneurysm should be indicated in adults with ascending aortic size of greater than $55 \mathrm{~mm}$ for degenerative aneurysms. For patients with BAV disease, surgical intervention should be considered if the size is greater than $45 \mathrm{~mm} .^{22}$

According to Sievers, ${ }^{20}$ the threshold size is related to body surface area, age, and pathology of the aortic wall. However, all the abovementioned recommendations were drawn from data of patients in the Western world, and it is known that the body surface area of the normal adult is smaller in Asian than in the Western world. Therefore we hypothesize that a smaller threshold diameter would be more appropriate in Asian patients. In our institute we made the decision for surgical intervention when the diameter of the ascending aorta reached $50 \mathrm{~mm}$ for degenerative aneurysms and $40 \mathrm{~mm}$ for BAV disease.
To the question of repair or replacement for a dilated ascending aorta, Robicsek and associates ${ }^{1}$ pointed out that patients with a diameter of greater than $6.0 \mathrm{~cm}$ should not undergo reduction aortoplasty. However, we consider the quality of the ascending aortic wall more than size as a determinant for the choice because the oversized ascending aorta could be reduced to normal range by aortoplasty if only the quality (including thickness and pathology) of the aortic wall is acceptable. In the current study the preoperative diameter of the ascending aorta was not significantly predictive of late death in the 2 groups according to the analysis of risk factors related to late death.

Although there was no difference in terms of postoperative events, shorter cardiopulmonary bypass time and aortic crossclamp time and fewer red blood cells were used in patients undergoing RRA than in patients undergoing AAR, reflecting the decreased technical complexity of the former procedure. This will result in an improved preservation for the myocardium and other organs, which can be advantageous for patients with high operative risks and when concomitant procedures are required. This result indicates that in this situation patients can benefit from RRA more than from AAR.

According to recent studies, the perioperative mortality of AAR and RRA was about $3 \%{ }^{23,24}$ and $0 \%$ to $1.5 \%,{ }^{2,15,17}$ respectively. There was no perioperative death in the current cohort, and it should be attributed to the 3 following factors: relatively young patients, only AVR as a concomitant procedure, and absence of aortic dissection or Marfan syndrome in the present study.

Consistent with the previous studies, ${ }^{15-17}$ there was no late redilatation of the ascending aorta after repair or replacement during follow-up in the present study. Moreover, we further focused on monitoring of the diameters of these 2 locations continuously. Our results show that both procedures result in good late outcomes without significant redilatation at the sinuses or proximal aortic arch. All diameters measured by means of computed tomographic analysis were within the acceptable range, and no reoperation was required, even in patients with BAV, which is defined as a structural disorder of the aortic wall. We attribute this to the decrease of tension in the aortic wall, which resulted from the reduction in internal diameter caused by the operation, according to the law of LaPlace. This result suggests that the fusiform ascending aorta with BAV is not a contraindication for RRA or AAR.

Therefore we recommend that for the treatment of fusiform ascending aortic aneurysm, RRA should be more frequently considered if only the quality of the aortic wall is acceptable.

\section{Study Limitations}

There are 2 limitations in this comparative study, which are the lack of prospective design and that the cases are 
not distributed in a random way. The bias of patient selection existed as a result of the respective indications of the 2 procedures. Because of the relatively strict indication for surgical procedures, a rigorous randomization in this kind of study might compromise the patients and therefore be impractical to carry out. For this reason, we believe that the current study is the most practical approach for comparison of the 2 surgical options at the present time.

\section{References}

1. Robicsek F, Cook JW, Reames MK Sr, Skipper ER. Size reduction ascending aortoplasty: is it dead or alive? J Thorac Cardiovasc Surg. 2004;128:562-70.

2. Bauer M, Pasic M, Schaffarzyk R, Siniawski H, Knollmann F, Meyer R, et al. Reduction aortoplasty for dilatation of the ascending aorta in patients with bicuspid aortic valve. Ann Thorac Surg. 2002;73:720-4.

3. Robicsek F, Thubrikar MJ. Conservative operation in the management of annular dilatation and ascending aortic aneurysm. Ann Thorac Surg. 1994;57:1672-4.

4. Egloff L, Rothlin M, Kugelmeier J, Senning A, Turina M. The ascending aortic aneurysm: replacement or repair? Ann Thorac Surg. 1982;34:117-24.

5. Kuralay E, Demirkilic U, Ozal E, Oz BS, Cingöz F, Günay C, et al. Surgical approach to ascending aorta in bicuspid aortic valve. J Card Surg. 2003;18:173-80.

6. Mueller XM, Tevaearai HT, Genton CY, Hurni M, Ruchat P, Fischer AP, et al. Drawback of aortoplasty for aneurysm of the ascending aorta associated with aortic valve disease. Ann Thorac Surg. 1997;63:762-6.

7. Jault F, Nataf P, Rama A, Fontanel M, Vaissier E, Pavie A, et al. Chronic disease of the ascending aorta. Surgical treatment and long-term results. J Thorac Cardiovasc Surg. 1994;108:747-54.

8. Sundt TM 3rd, Mora BN, Moon MR, Bailey MS, Pasque MK, Gay WA Jr. Options for repair of a bicuspid aortic valve and ascending aortic aneurysm. Ann Thorac Surg. 2000;69:1333-7.

9. Immer FF, Barmettler H, Berdat PA, Immer-Bansi AS, Englberger L, Krahenbuhl ES, et al. Effects of deep hypothermic circulatory arrest on outcome after resection of ascending aortic aneurysm. Ann Thorac Surg. 2002;74:422-5.

10. Simon-Kupilik N, Schima H, Huber L, Moidl R, Wipplinger G, Losert U, et al. Prosthetic replacement of the aorta is a risk factor for aortic root aneurysm development. Ann Thorac Surg. 2002;73:455-9.
11. Scharfschwerdt M, Sievers HH, Greggersen J, Hanke T, Misfeld M. Prosthetic replacement of the ascending aorta increases wall tension in the residual aorta. Ann Thorac Surg. 2007;83:954-7.

12. Moon MC, Morales JP, Greenberg RK. The aortic arch and ascending aorta: are they within the endovascular realm? Semin Vasc Surg. 2007;20:97-107.

13. Cohen O, Odim J, De la Zerda D, Ukatu C, Vyas R, Vyas N, et al. Long-term experience of girdling the ascending aorta with Dacron mesh as definitive treatment for aneurysmal dilation. Ann Thorac Surg. 2007;83:780-4.

14. Walker T, Bail DH, Gruler M, Vonthein R, Steger V, Ziemer G. Unsupported reduction ascending aortoplasty: fate of diameter and of Windkessel function. Ann Thorac Surg. 2007;83:1047-53.

15. Polvani G, Barili F, Dainese L, Topkara VK, Cheema FH, Penza E, et al. Reduction ascending aortoplasty: midterm follow-up and predictors of redilatation. Ann Thorac Surg. 2006;82:586-91.

16. Feindt P, Litmathe J, Börgens A, Boeken U, Kurt M, Gams E. Is size-reducing ascending aortoplasty with external reinforcement an option in modern aortic surgery? Eur J Cardiothorac Surg. 2007;31:614-7.

17. Arsan S, Akgun S, Kurtoglu N, Yildirim T, Tekinsoy B. Reduction aortoplasty and external wrapping for moderately sized tubular ascending aortic aneurysm with concomitant operations. Ann Thorac Surg. 2004;78:858-61.

18. Carrel T, von Segesser L, Jenni R, Gallino A, Egloff L, Bauer E, et al. Dealing with dilated ascending aorta during aortic valve replacement: advantages of conservative surgical approach. Eur J Cardiothorac Surg. 1991;5:137-43.

19. Tadros TM, Klein MD, Shapira OM. Ascending aortic dilatation associated with bicuspid aortic valve: pathophysiology, molecular biology, and clinical implications. Circulation. 2009;119:880-90.

20. Sievers HH. Reflections on reduction ascending aortoplasty's liveliness. J Thorac Cardiovasc Surg. 2004;128:499-501.

21. Elefteriades JA. Natural history of thoracic aortic aneurysms: indications for surgery, and surgical versus nonsurgical risks. Ann Thorac Surg. 2002;74(suppl): S1877-80.

22. Borger MA, Preston M, Ivanov J, Fedak PW, Davierwala P, Armstrong S, et al Should the ascending aorta be replaced more frequently in patients with bicuspid aortic valve disease? J Thorac Cardiovasc Surg. 2004;128:677-83.

23. Achneck HE, Rizzo JA, Tranquilli M, Elefteriades JA. Safety of thoracic aortic surgery in the present era. Ann Thorac Surg. 2007;84:1180-5.

24. Shapira OM, Aldea GS, Cutter SM, Fitzgerald CA, Lazar HL, Shemin RJ Improved clinical outcomes after operation of the proximal aorta: a 10-year experience. Ann Thorac Surg. 1999;67:1030-7. 